\title{
Investigation on Traffic Assignment of City Center Area Based on Improved Logit Route Choice Method
}

\author{
Deng Hai Long ${ }^{1, a}$,Xu Mei Lin ${ }^{2, b}$ and GaoJian Zhi ${ }^{3, c *}$ \\ ${ }^{1}$ CCCC Second Highway Consultants Co.Ltd, Wuhan, Hubei, P.R.China \\ ${ }^{2}$ Huazhong University of Science and Technology, Wuhan, Hubei, P.R.China \\ ${ }^{3}$ Huazhong University of Science and Technology, Wuhan, Hubei, P.R.China \\ a1067858805@qq.com, b2546558938@qq.com, c252793255@qq.com
}

Correspondence should be addressed to Gao Jian Zhi (252793255@qq.com)

\begin{abstract}
Keywords:Logit method, Route choice, Road resistance function, Traffic assignment
Abstract.With the development of social economy, the problems of urban traffichas become increasingly prominent. It is necessary to pay attention to the traffic planning. Traffic assignment is an important content of traffic planning. The route choice of the traveler is the focus of traffic assignment. In the study of route selection, the most commonly used method is Logit route choice. However, in the macro-level road network, Logit route choice method has the problems of exhaustive path and complicated calculation process. The road network in the urban center belongs to the meso-level road network and the traffic characteristics are more consistent. It is of significance to investigate the traffic assignment in the urban center by using Logit routechoice method.

In this paper, based on the framework of the optimal path evaluation index system, the method of Analytic Hierarchy Process (AHP) is proposed to improve the calculation method of path flowin traditional Logit route choice. First, according to the influence of the road hierarchy on the route choice, the road impedance function with the road hierarchy consideration is established. Then, the method of calculating the flow distribution rate in traditional Logit route choice is improved by using the road resistance function of city center area previously established, and the traffic assignment method of the urban central area is constructed by combining the successive equilibrium algorithms in the multi-path allocation method. Finally, the case analysis of Zone F traffic flow distribution is carried out, and the more reasonable distribution result is found out. The results provide a scientific basis for the management and planning of the traffic department.
\end{abstract}

\section{Introduction}

In the context of rapid urban development, traffic congestion has become increasingly prominent. One of the ideas to solve this problem is to improve the accuracy of demand forecasting and ensure the balance between supply and demand. Traditional Logit route choice has two major drawbacks [1] [2]. On the one hand, it is assumed that each route is independent of each other, whichcannot reflect the independence of overlapping sections. On the other hand, the probability of selection is only determined by the absolute difference between the impedances of each path, which is not in accordance with the actual situation.

In order to improve the accuracy of demand forecasting, this paper investigates the process of traveler route choice. By constructing the road impedance function with the road hierarchy consideration, and taking the optimal path evaluation index system of AHP as the frame, the method of calculating the traffic distribution rate of the traditional Logit route choice is improved. The 
result illustrates that traffic distribution in the city center area fits the actual travel.

\section{Improvement of Logit Route Choice Method in Urban Center Area by Considering the Influence of Road Hierarchy}

Establishment of travel route choice process

The traffic in the urban center area has the characteristics of double peaks in morning and evening, and there is a centripetal distribution pattern in space. Logit route choice can be used to describe the distribution of traffic flow in the center area, avoiding the unreasonable phenomenon that the traffic is completely concentrated on a certain path. By employing AHP and the evaluation system of optimal path [3], the travel route choice behavior is systematically analyzed in urban center, meanwhile, the factors influencing travel are summed up as four items including travel time, travel distance, comfort, and safety. Different weights are assigned to the four influencing factors and a comparison matrix is set up. By integrating the actual impedance and random utility in the perceived impedance, the weight model is constructed.

Calculation of Route Choice Indicator Attribute

Because of the inconsistency between the dimensions and scales of the various attributes, it is necessary to make the factors dimensionless. Travel distance and travel time are classified as cost attributes. The smaller the attribute value of attributes in this category, the greater the contribution to the route traffic allocation rate. Security and comfort are classified as efficiency attributes. The greater the attribute value of attributes in this category, the greater the contribution to the traffic distribution rate of the route. The normalized functions of cost-benefit attributes are as follows:

$$
\begin{aligned}
& Q_{i}=\left\{\begin{array}{cl}
1 & \left(x_{i}=D_{i}\right) \\
\frac{x_{i}-d_{i}}{D_{i}-d_{i}} & \left(d_{i}<x_{i}<\mathrm{D}_{i}\right) \\
0 & \left(x_{i}=d_{i}\right)
\end{array},(2-1)\right. \\
& Q_{i}=\left\{\begin{array}{cl}
1 & \left(x_{i}=D_{i}\right) \\
\frac{D_{i}-x_{i}}{D_{i}-d_{i}} & \left(d_{i}<x_{i}<\mathrm{D}_{i}\right) \\
0 & \left(x_{i}=d_{i}\right)
\end{array}\right.
\end{aligned}
$$

Where $Q_{i}$ is the normalized attribute value, $x_{i}$ is the original attribute value and $\left[d_{i}, D_{i}\right]$ is the original attribute value range.

Construction of Route Choice Method

Calculation steps are as follows:

(1) To determine the route choice hierarchy, the target layer $\mathrm{Z}$ is the travel route choice, while the guideline layer $\mathrm{C}$ includes the path distance, travel time, comfort and security.

(2) To establish the judgment matrix of criterion $\mathrm{C}$ in the optimal path evaluation system. Under the same scale, the uniform matrix method is used to sort the hierarchy, and the grade is evaluated according to its importance. With Saaty's table of importance [4], the scale of each factor 
is listed, and the judgment matrix of the four influencing factors on the target layer $\mathrm{Z}$ is constructed, then the consistencyshould be tested. The judgment matrix are as follows:

$$
\begin{gathered}
C_{1} \\
A=C_{2} \\
C_{1} \\
C_{2} \\
C_{3} \\
C_{4}
\end{gathered}\left[\begin{array}{llll}
a_{11} & a_{12} & a_{13} & a_{14} \\
a_{21} & a_{22} & a_{23} & a_{24} \\
a_{31} & a_{32} & a_{33} & a_{34} \\
a_{41} & a_{42} & a_{43} & a_{44}
\end{array}\right] .
$$

Where, $C_{1}, C_{2}, C_{3}, C_{4}$ indicates path distance,travel time, comfort,saftyin the guideline layer, respectively, $a_{i j}$ indicates the mutual comparison coefficient of each factor in the criterion layer $\mathrm{C}$ to the target layer $Z$. The vector of weights is calculated using the arithmetic mean of the column vectors, which can be expressed as:

$$
A_{i}=\sum_{j=1}^{n} \frac{a_{i j}}{\sum_{k=1}^{n} a_{k j}} \cdot(2-3)
$$

(3)To calculate the attribute value of the influencing factors in each scheme of the program level $\mathrm{P}$ and carry out the dimensionless calculation.

The path distance is determined according to the actual road length in each travel; the travel time is computed by the road impedance function in the center area. The road comfort and safety can be determined according to the road facility grade and $\mathrm{V} / \mathrm{C}$ ratio. When the travel path contains different types of roads, the values of the travel distance and travel time can be obtained by the equation (2-3). The comfort and safety attributes in the travel route can be calculated according to equation (2-4):

$$
\begin{aligned}
& Q_{i j}=\sum_{a=1}^{n} q_{i a},(2-4) \\
& Q_{i j}=\sum_{a=1}^{n} q_{i a} \times \frac{l_{a}}{\sum_{a=1}^{n} l_{a}} .
\end{aligned}
$$

Among them, i,j,a, 1 represent the influencing factor number, the jth travel path, the ath road in the jth travel path, the length of the link, respectively. Finally, the dimensionless attribute value of each program's influencing factors is obtained.

(4) To construct road traffic flow distribution rate at all levels.

By using the weight vectors $A_{i}$ calculated from equation (2-2) and (2-3) and the attribute values of the dimensionless factors $Q_{i j}$, the traffic distribution rate of route is constructed. It can be 
described as:

$$
P_{j}=\frac{\sum_{i=1}^{n} Q_{i j} \times A_{i}}{\sum_{j=1}^{n} \sum_{i=1}^{n} Q_{i j} \times A_{i}} \cdot(2-6)
$$

Where $P_{j}$ indicates the probability of the traveler selecting the jth route, and the greater the value of $P_{j}$, the more travelers prefer to select this route.

By utilizing successive averaging algorithm [5] and combining with the improved Logit path selection method, a traffic assignment method is constructed.

The improved logit route choice method simplifies the calculation method of the perceived impedance in the traditional logit route choice method. AHP is used to calculate the weight of four factors in the route choice process. The method combines the actual resistance and the random utility in the perceived impedance, not only weakening the influence of the random utility in calculating the flow distribution rate of the path, but also avoiding that the probability of path selection is only determined by the absolute difference between the impedances of paths.

\section{Construction of the Road Resistance Function in Urban Center Area}

The study on the resistance function is mainly based on the BPR function of the US Federal Highway Administration [6]. In order to reflect the characteristics of road traffic flow more accurately, it is proposed to set up the road resistance function considering the road hierarchy.

3.1 The Road Resistance Function of Expressway

The impedance of the urban expressway is mainly concerned with the delay of the basic segmentand the node. The latter is the delay in the interweaving area. It can be expressed as:

$$
t_{i j}=d_{i j}+y \cdot(3-1)
$$

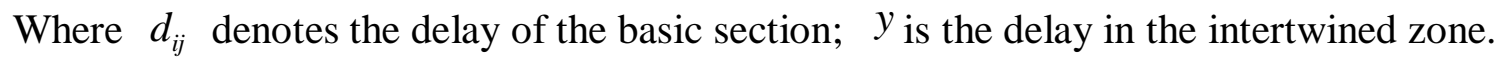

The BPR function can be used to express the delay of basic segment:

$$
d=t(0)\left[l+\alpha\left(\frac{q}{e}\right)^{\beta}\right] .(3-2)
$$

By introducing the basic segment length 1 and the design speed $\mathrm{v}$ of road, the impedance function can be expressed as:

$$
d=\frac{l}{v}\left[1+\alpha\left(\frac{q}{e}\right)^{\beta}\right]
$$


When calculating the delay in the interweaving area, it is assumed that the vehicle maintains a constant speed when entering and exiting the right-hand ramp, the speed is less than that of the main stream flow, supposed that only the outermost lane flow is disturbed by the vehicles entering and exiting. When the expressway traffic is affected by the intertwined zone, in the first stage, affected by the import and export vehicles on the right side, vehicles on the main road decelerate; in the second stage, vehicles on the main road decelerate to the following speed and follow the vehicles. The car-following speed is the speed of vehicles on auxiliary lane. Impact factor $\gamma$ is proposed for analysis. When there is no impact on the right side, the speed is $v_{1}$, and time is $t_{1}$; otherwise, the speed is $v_{2}$, andtime is $t_{2}$. According to kinematics, the delay impact $\Delta \mathrm{t}$ is described as:

$$
\Delta t=t_{2}-t_{1}=L \times\left(\frac{1}{\bar{v}}-\frac{1}{v_{1}}\right)
$$

Where ${ }^{\bar{v}}$ represents the average vehicle speed when there is a right-side inlet, L represents the length of affected road.

According to the definition of the basic road segment and the influential segment of urban expressways,

$$
\Delta t=\frac{5\left(v_{1}-v_{2}\right)}{6 v_{2}} t_{1} \cdot(3-5)
$$

According to the study of Chen et al. [7], there exists the following relationship between traffic speed and road traffic:

$$
V=\frac{V_{f}}{1+(q / e)} \cdot(3-6)
$$

Where $V_{f}$ is unobstructed speed, $q / e$ is the ratio of road flow and capacity.Thus,

$$
\Delta t=\frac{5}{6} \times\left|\left(\frac{q_{1}-q_{2}}{e+q_{1}}\right)\right| \times t_{1} \cdot(3-7)
$$

Where $q_{1}, q_{2}$-the traffic flow under the speed $v_{1}, v_{2} ; t_{1}$ - the driving time without the effect of interweaving area, $t_{1}=\mathrm{L} / \mathrm{v}$.Let 
$\gamma=\frac{5}{6}\left|\left(\frac{q_{1}-q_{2}}{e+q_{1}}\right)\right| \cdot(3-8)$

The travel delay in the intertwined zone of expressway is:

$y=n \times \gamma \times t_{1} \cdot(3-9)$

Where $n$ is the number of interweaving areas in urban expressway.

As a result,the road resistance function of freeway in the center area is

$$
t_{i j}=\frac{l}{v}\left[1+\alpha\left(\frac{q}{e}\right)^{\beta}\right]+\left[n \times \frac{5}{6}\left|\left(\frac{q_{1}-q_{2}}{e+q_{1}}\right)\right| \times \frac{L}{v}\right] .(3-10)
$$

\subsection{The Road Resistance Function of Arterials}

The road resistance function of arterials is divided into segment impedance and node impedance. Based on the BPR function, the segment impedance is added to the delay of bus, while delays caused by flow of motor and non-motorized vehicles will be considered in a few arterials without non-physical medians [8]. The node impedance mainly includes the delay of signal control intersections. By using Webster formula t, the delay of signalized intersection can be expressed as:

$$
x=\frac{T_{c}(1-\lambda)^{2}}{2(1-\lambda c)}+\frac{c^{2}}{2 q(1-c)}-0.65\left(\frac{T_{c}}{q^{2}}\right)^{1 / 3} c^{(2+5 c)} .
$$

Where $T_{c}$ is cycle time, $\lambda$ is split, $q$ denotes flow, $c$ denotes saturation.

As a result, the road resistance function of arterial road in the center area is

$$
t_{i j}=\left\{\frac{l}{v}\left[1+\alpha\left(\frac{q+q_{g}}{e}\right)^{\beta}\right]+\bar{t}_{1} \frac{t_{g} q_{g}}{3600}\right\}+\left[\frac{T_{c}(1-\lambda)^{2}}{2(1-\lambda c)}+\frac{c^{2}}{2 q(1-c)}-0.65\left(\frac{T_{c}}{q^{2}}\right)^{1 / 3} c^{(2+5 c)}\right]
$$

Where $q_{g}$ is the flow of bus, $t_{g}$ is the travel time of a bus on the road, $\bar{t}_{1}$ is the average delay of the social vehicles caused by a bus.

3.3 The Road Resistance Function of Minor Arterials and Locals

Due to the non-physical medians of minor arterials and locals, the road impedance needs consider non-motorized vehicles' delay [9].

$$
d_{i j}=\frac{l}{v}\left[1+\alpha\left(\frac{q}{e}\right)^{\beta}\right]+F .
$$


Then the non-motorized delay item $F$ is discussed. The delays caused by non-motorizedvehicles can be divided into three parts: first, the delay of deceleration caused by the non-motorized vehicles' interference; second, the follow-up delay caused by maintaining a safe distance; third, the acceleration delays caused by the motorized vehicle when a non-motorized vehicle leaves.

$$
F=d_{d}+d_{f}+d_{a}
$$

Where $d_{d}$ is deceleration delay; $d_{f}$ is follow-up delay; $d_{a}$ is acceleration delay.

$$
d_{d}=d_{\mathrm{a}}=\frac{\left(v_{1}-v_{2}\right)^{2}}{2 b \times v_{1}} \text {. }
$$

Where $v_{1}$ is normal driving speed; $v_{2}$ is speed after deceleration; $\mathrm{b}$ stands for acceleration and deceleration.

$$
d_{f}=\frac{\left(v_{1}-v_{2}\right)(\lambda-\mu)}{v_{2} \times \lambda} \times t
$$

Where $\lambda$ is average arrival rate for non-motorized vehicles; $\mu$ is average departure rate for non-motorized vehicles; $t$ is the time of non-motorized vehicles occupying the driveway (6s). Thus,

$$
F=\frac{e v_{f}(a+b)}{2 a b} \times \frac{\left(q_{1}-q_{2}\right)^{2}}{\left(e+q_{1}\right)\left(e+q_{2}\right)^{2}}+\left(\frac{q_{2}-q_{1}}{e+q_{1}}\right) \times \frac{\lambda-\mu}{\lambda} \times t
$$

Where $a$ is the acceleration of a motorized vehicle from a non-motorized vehicle; $e$ is road capacity. $b$ is the deceleration of a motorized vehicle under the influence of a non-motorized vehicle. ${ }^{q_{1}, q_{2}}$ are the flow of motorized vehicles before and after the impact of a non-motorized vehicle.

The node impedance of minor arterials and locals can be calculated according to Webster's formula of the equation (3-12). In summary, according to equation (3-14), (3-13) and (3-17), the expression of road resistance function of minor arterials and locals can be obtained.

Road resistance function in the urban center area is mainly used in the calculation of the travel time in the improved logit route choice method, which will be more reasonable description of the delay of the vehicle during the trip at different grades of roadways, fully embodying the impact of the road hierarchy on the choice of travel route. 


\section{Case Analysis - Traffic Flow Distribution in Zone F}

Regional Road Network and Traffic Zone

The layout of the road network in zone $\mathrm{F}$ shows the shape of the " $\mathrm{T}$ " with the main skeletons of two expressways (east-west and north-south). The area is divided into 10 traffic zones and 8 peripheral zones.

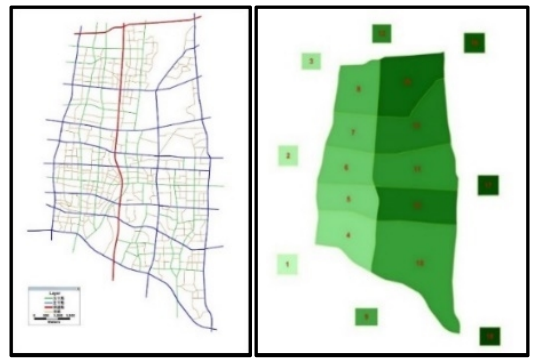

Fig. 4-1 Road network of zone F
Fig. 4-2 Traffic zones of zoneF

Traffic Assignment Based on Improved Logit Route Choice Method

The traffic demand prediction is based on the traditional four-stage method. Before carrying out the process of traffic assignment in the $\mathrm{F}$ area, we use the traditional traffic generation and distribution method to get the data of traffic assignment. Since there are two travel peaks in the central region of the city, the early morning peak period of the F area is selected for the study. Resident trip generation and full-mode travel expectation line of $\mathrm{F}$ are as follows.

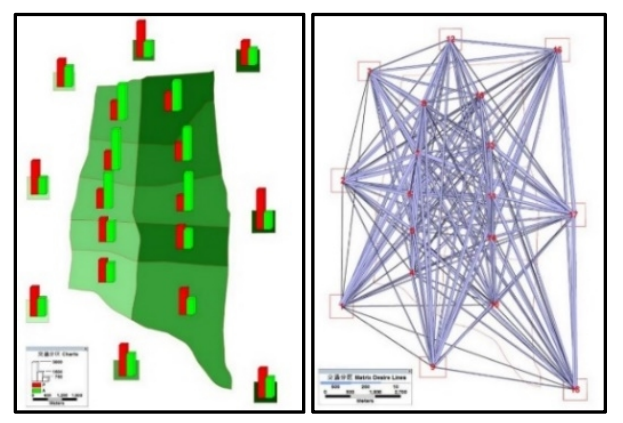

Fig. 4-3 Trip generation of zone F Fig. 4-4 Expectation line map of zone F

The traffic distribution method based on conventional Logit route choice and improved Logit route choice are used for traffic assignment, respectively. A custom volume delay function (VDF) is created in the traffic simulation software TransCAD. After the VDF has been created, the command of "Build-Build Solution" is used in Visual Studio. Started TransCAD with Visual Studio's command of "Debug-Start", the custom VDF and the successive averaging algorithm in the multipath allocation method are used for traffic assignment. The result is shown below.

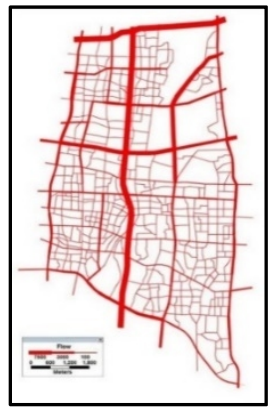

Fig. 4-5 Results of general logit route choice assignment

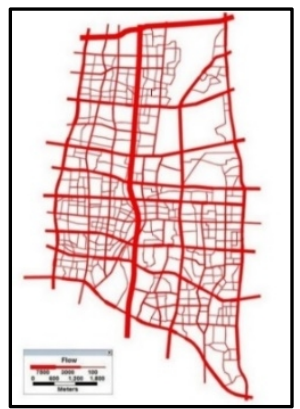

Fig. 4-6 Results of improved logit route choice assignment 


\section{Comparison and Analysis}

By comparing Figure 4-5 with Figure 4-6, it can be found out:

1.When using the improved logit route choice method for traffic assignment, the traffic volume distribution on the entire road network is more even and the traffic on some main arterials is increased, compared with the allocation result calculated by the conventional logit route choice algorithm.

2. When using the improved logit route choice method, the mainstream of traffic flow is consistent with the distribution results calculated by the conventional logit path algorithm. However, the flow of the entire road network has shifted to some extent. The traffic volume of some major arterials has been transferred to the minor arterials and locals.

3.Traffic demand is always fixed for the entire city, so the allocation method will not have an impact on the total traffic demand. The change of the calculation method of the road resistance function affects the traffic distribution rate, which makes the choice of the route changed when the vehicle is distributed.

\section{Conclusion}

In this paper,on the one hand, the road impedance functions of different grade are proposed,because there are different factors ofroad impedance function.major arterials, minor arterials and locals will be affected by weaving area, buses and non-motorized vehicles, respectively.On the other hand, by considering the selection index in the process of route choice, the analytic hierarchy process (AHP) is used to integrate the actual impedance and random utility term in the perceived impedance. The calculation of road traffic distribution rate in logit route choice method is improved, which avoids that the probability of route choice is only determined by the absolute difference of impedances between the paths.Finally, based on the improved logit routec choice method, the research on road network $\mathrm{F}$ distribution is carried out. The results show that when using the improved logit route choice method to allocate flow, the distribution results are better than the traditional logit model. Traffic is exchanged more frequently between roads, and traffic is more evenly distributed across all levels of roads. This is because the improved logit route choice method does not merely refer to the travel time as a reference when determining the traffic distribution rate. The path length distribution and the path comfort factor are also taken into account.

\section{Acknowledgements}

The work described in this paper was supported by the Innovation Research Fund (HUST:2016YXMS100).

\section{Literature References}

[1] Bell, M.G.H.: Alternatives to Dial's Logit Assignment Algorithm. Transportation Research Part B Methodological, Vol.29(1995), p. 287-295.

[2] Xuefei Li:Research on traffic assignment model and algorithm based on extended Logit.Beijing:Beijing Jiaotong University(2014).

[3]Yan Sun:Optimal Route Selection Based on Gray System Theory.Journal of Civil Engineering. Vol.01(2003),p94-98. 
[4] Saaty, Thomas L.:Prediction, Projection And Forecasting : Applications Of The Analytic Hierarchy Process In Economics, Finance, Politics, Games and Sports / Thomas L. Saaty, Luis G. Vargas. (1991).

[5]Powell W B, Sheffi Y. The Convergence of Equilibrium Algorithms with Predetermined Step Sizes. Transportation Science, Vol.16(1982), p45-55.

[6] Transportation Research Board Business Office. Highway Capacity Manual, Washington DC(2000), p. 98-112.

[7]Sheng Chen:Study on Practical Relationship Model of Urban Road Traffic Flow Velocity.Nanjing :Nanjing University(2004).

[8]Xianghai Meng, Shilian Li:Study on the Resistance Function of Expressway and Trunk Road in Big Cities.Transportation System Engineering and Information.Vol.04 (2005),p.31-34.

[9]Mingwen Wang: Research on the Impact of Non - motorized Vehicles on Traffic Flow.Beijing :Beijing industry university(2004). 